EPiC Series in Engineering
Volume 3, 2018, Pages 274-281
HIC 2018. 13th International
Conference on Hydroinformatics

\title{
Hydraulic model calibration of pressure reduced zones with multiple input valves
}

\author{
Attila Bibok ${ }^{1}$, Roland Fülöp ${ }^{1}$ \\ ${ }^{1}$ Budapest University of Technology and Economics, Müegyetem rkp $3 \mathrm{kmf} 45$, \\ Budapest H-1111, Hungary
}

Corresponding author: bibok.attila@epito.bme.hu

\begin{abstract}
Pressure management is a widely adopted technique to decrease background leakage or to extend the lifespan of the pipe network [1]. In some cases, it is inevitable to deploy multiple pressure reducing valves to supply a particular zone. In order to supply water to the customers with optimal pressure head, the precise setting of the parallel pressure reducing valves' (PRV) target pressure is required. Steady-state hydraulic models like EPANET has the functionality to simulate pressure loss of a pressure-reducing valve [1]. This can be simulated by adding minor-loss after the pipe, or by modifying the properties of the next link on the downstream side. Either way, the proper setting of the coefficients is essential to calibrate the hydraulic model. In this paper, two non-linear optimization methods were utilized to calibrate the hydraulic model with multiple input values.
\end{abstract}

\section{Introduction}

The pressure in drinking water distribution systems are usually in between 1.5 and 6.0 bar to provide sufficient pressure, but still so high that could damage the consumers' domestic system or appliances. In order to comply utilities has to form pressure-reduced zones or reduce the overall pressure in a zone if it is allowed by the lower boundary. In some cases, a single pipe doesn't have the capacity to deliver all the flow of the zone with the reduced pressure. To overcome this issue, multiple inputs by PRV's can be a solution, which induces new engineering problems, for example, the proper setting of the valves. If the valves are not set to the same target head, some valves might be closed unless peak demand occurs, which causes a change in flow direction. Thus, the probability of turbidity problems increases if sedimentation is present in the distribution network. The variability of downstream pressure is about $\pm 0.2-0.3$ bar [3] even when the valve size is designed properly. Based on the pressure measurements of Budapest Waterworks, this variability can be as high as \pm 0.6 bar (Figure 1). This phenomenon is only present in zones where more than one pressure reducing valve is supplying the same zone. Single input pressure reduced zones have stable pressure readings with \pm 0.1 bar or lower variability around the pressure setting (Figure 2), although time controlled pressure setting was applied and the peak flow rate was eight times higher than the minimum night-flow. 
In order to ensure that supplied pressure meets the pressure demand even at peak demand scenarios, it is necessary to calibrate the hydraulic model to the pressure and flow measurements at the input points. The hydraulic model was built in EPANET, which can be considered as an unofficial industrial standard due to its adaptation all around the world.

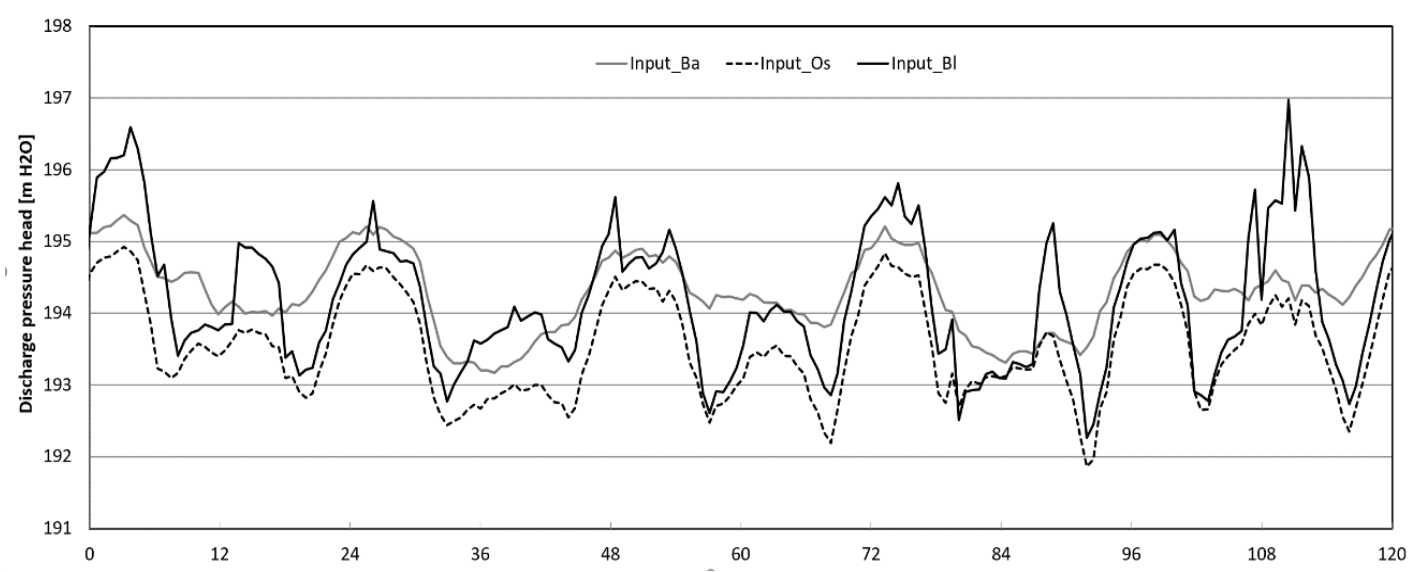

Figure 1: Pressure readings in a multiple input pressure reduced zone

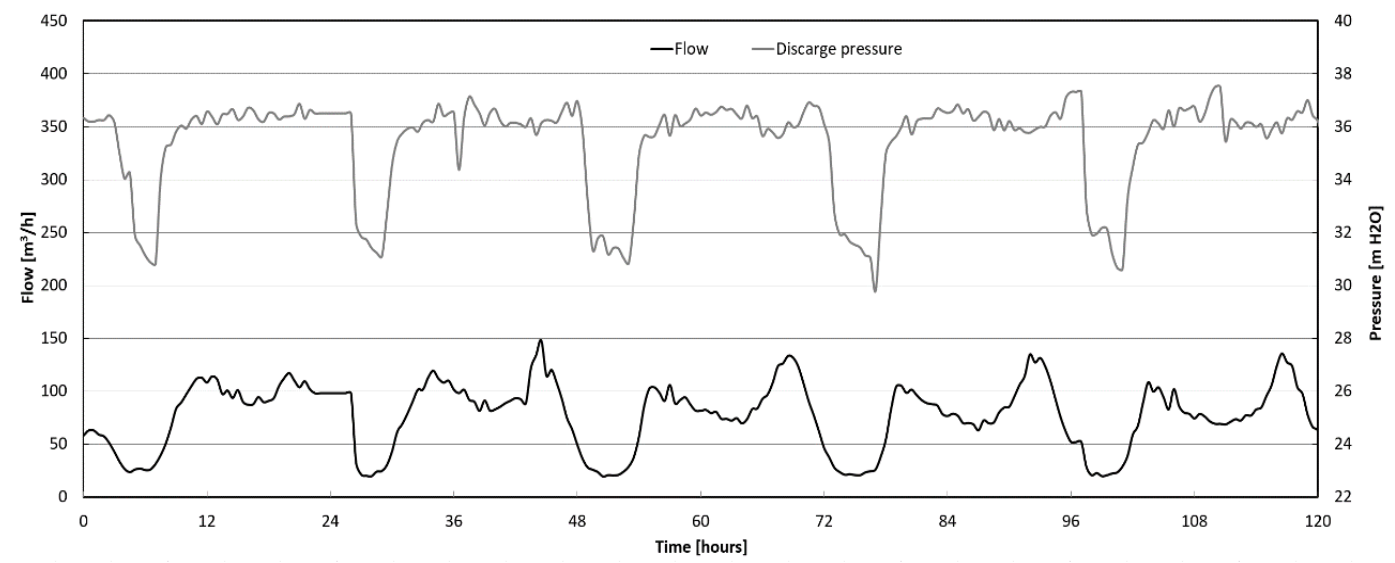

Figure 2: Pressure and flow readings in a single input pressure reduced zone

\section{Methodology}

A five-day-long period of continuous flow and pressure measurement time-series were used for calibration purposes. This length ensures the generality of the calibrated model. One flow meter and one pressure meter on the suctions side are available. Both the pressure and flow measurement time series represents half-hour mean values given in $\mathrm{m} \mathrm{H} 2 \mathrm{O}$ and $\mathrm{m}^{3} / \mathrm{h}$ units. Minor loss coefficient and valve target pressure were modified to fit the simulated flow and pressure measurement time-series. The flow and pressure values are highly sensitive to the pressure setting and loss coefficients so nonlinear optimization methods should be utilized to find the near best solution for this multiple-variable problem instead of manual calibration. EPANET-Matlab Toolkit is an open source wrapper for EPANET classes, which combines Matlab's flexibility, and computational abilities with EPANET's functionality[4]. 


\subsection{Optimization}

Two non-linear optimization methods in Matlab were utilized in the research. Fminsearch is an unconstrained non-derivative multivariable optimization method based on the Nelder-Mead Simplex Method [5]. The other method is called simulated annealing, which is a bound-constrained probabilistic method [6]. Because physical properties of the system constrain the values of the target pressures and the loss coefficients, tight boundaries might speed up the probabilistic optimization run of simulated annealing. According to the pressure measurements on the downstream sides, target heads have to be 157 to 166 meters over the level of the Baltic Sea.

\subsection{Pilot zone}

The pilot zone used for model calibration is located at the southeast side of Budapest. The network consisted of 1637 nodes, 2045 links and 3 inputs through PRVs (Figure 3). Annual billed consumption was the weight of demand allocation, which was summarized for every link. The boundary condition of pressure on the upstream side was given as a reservoir with a pattern based level.

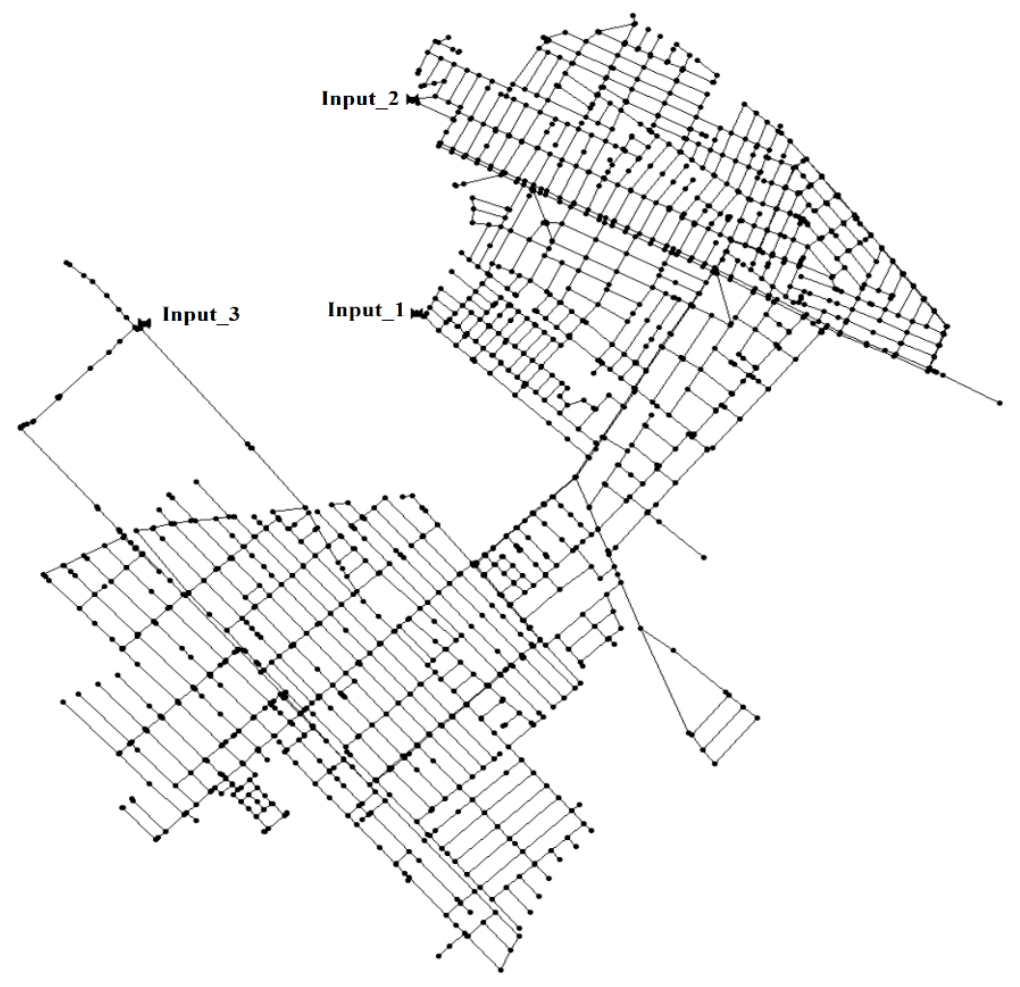

Figure 3 Network layout of the pilot zone

. It is essential to provide the most accurate demand allocation model to get system head curves for the input nodes that represent real conditions. The demand pattern was calculated by the sum of the three flow meters at the PRV's. The downstream pressure was set by PRVs.

\section{Results and discussion}

All of the used optimization methods were capable of calibrating the hydraulic model compared to the flow measurements. The ratio of input flows showed high correlation between the measured and the 
simulated values regardless of the optimization method. The error in flow rate on the second day is significant, although there was no irregular water consumption on that day, like flushing, fire-flow or burst on a main (Figure 4). The source of this phenomenon is that the generalized demand weights did not match the real demand distribution between the nodes for that day. The centre of demand is considered constant by using generalized demand weights, although in reality the centre of demand is moving depending on the actual demand distribution along the pressure zone.
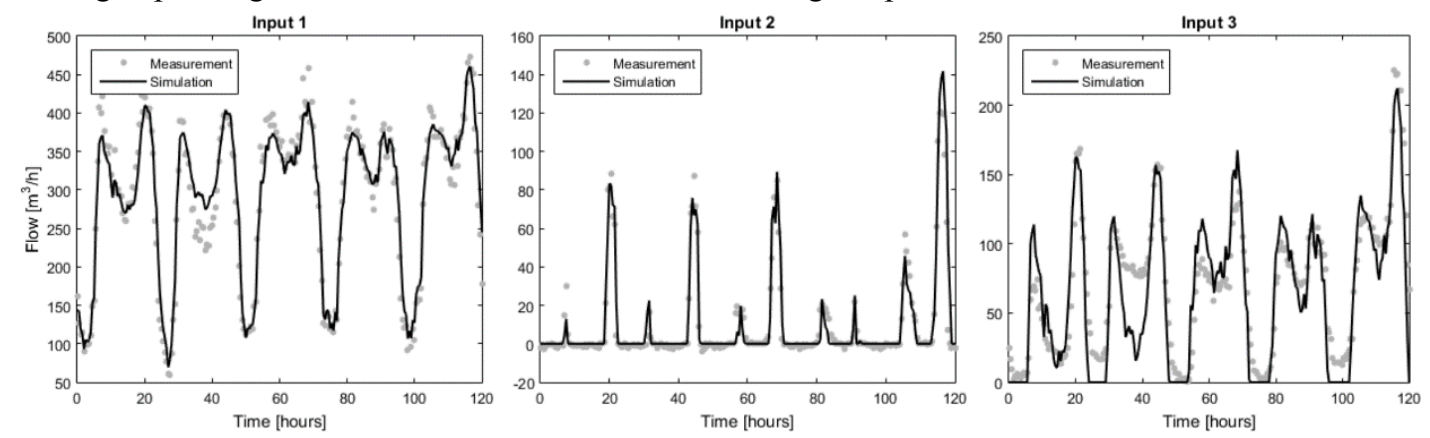

Figure 4 Calibration result in regard of flow measurements

The simplex method converged faster to a local minimum, but simulated annealing provided better fit after more iterations, due to the probabilistic approach it utilizes. Adding pressure measurement timeseries to the calibration was successful up to one pressure measurement (Figure 5). Otherwise, no optimization run could meet the stopping criteria even after more than 2000 iterations. Therefore, there is trade-off between the pressure measurement time-series or the physical model of pressure loss calculation in EPANET is not able to simulate the conditions observed in the field measurement datasets.
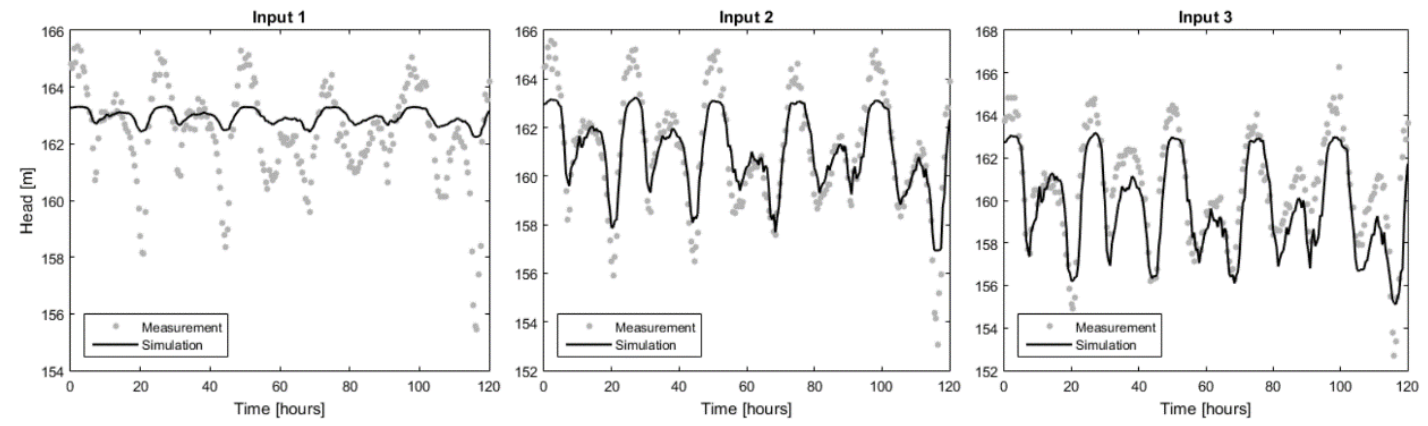

Figure 5 Calibration result in regard of pressure measurements

In order to overcome this issue, pressure meter reference error was added for every pressure measurement, increasing the number of variables to 9 . When simulated annealing was used, upper and lower boundaries for reference error were set to -2 to $+2 \mathrm{~m} \mathrm{H} 2 \mathrm{O}$. The higher number of variables reduced the performance of both methods. Displaying the operating point cloud of Input 1 showed that the relationship between the pressure loss and the flow rate of the PRV are cannot be described with a coefficient of a quadratic function, like minor loss coefficient [2, 7]. The normalized root-mean-squared error was $27 \%$ for the best fitting curve (Figure 6). The point clouds of the other inputs were not valid for this type of analysis, because the pressure setting was lower than Input 1, thus at minimum night flow, Input 1's downstream pressure influenced the pressure values at the other two inputs. 


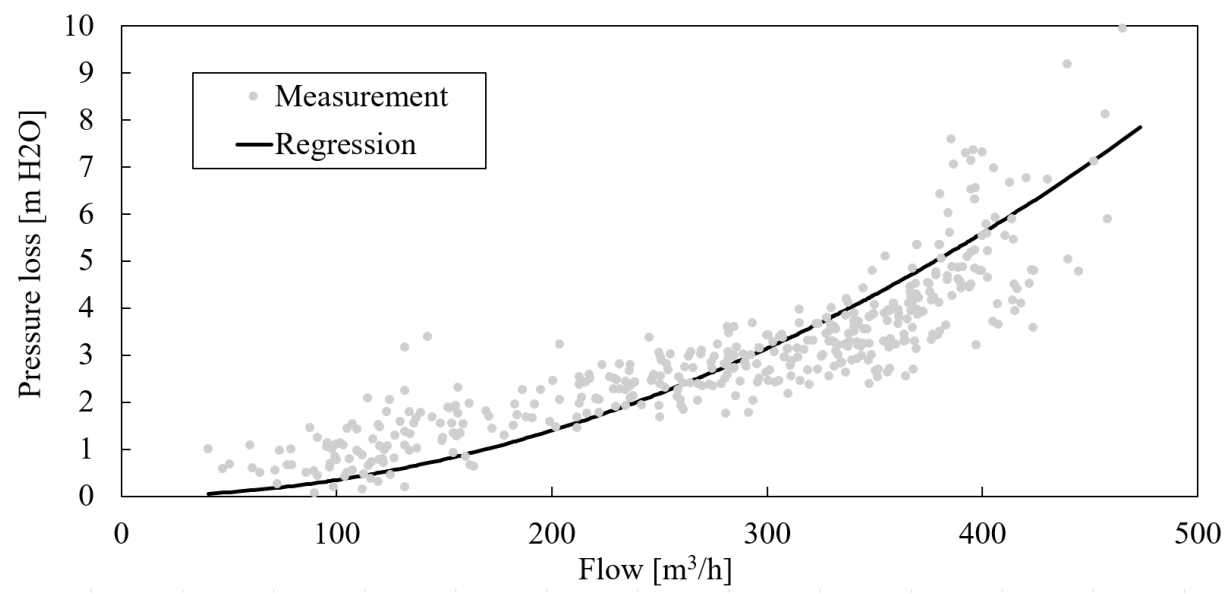

Figure 6 Flow - Pressure loss point cloud and best fitting quadratic regression

In order to overcome the limitation of quadratic minor headloss functions, the flow domain was divided into three sections at Input 1 (Figure 7) and into two sections at Input 3. It is possible to handle headloss on the downstream side as a function of flow by rule-based controls on parallel pipes after the PRV (Figure 8).

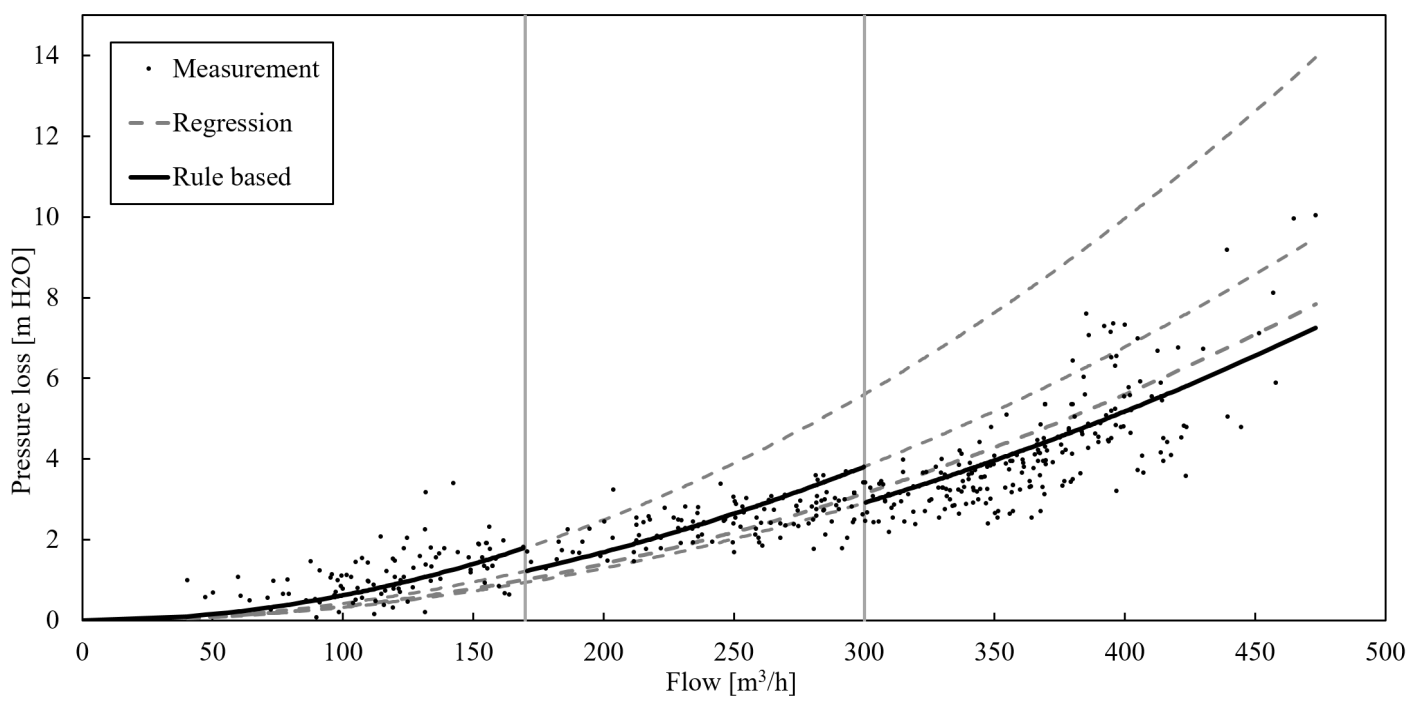

Figure 7 Modified flow-pressure loss curve by rule-based controls at Input 1

The pressure settings of the PRVs' were fixed and determined manually based on the maximum pressure values where flow was observed. The pressure setting of the valves was not changed and no time-based controls were applied in the 20 months period, which was used for analysis from 2016.01.01 to 2017.09.01. Input 1 and Input 3 were open most of the time, but Input 2 opened less frequently, mostly at peak demands. 


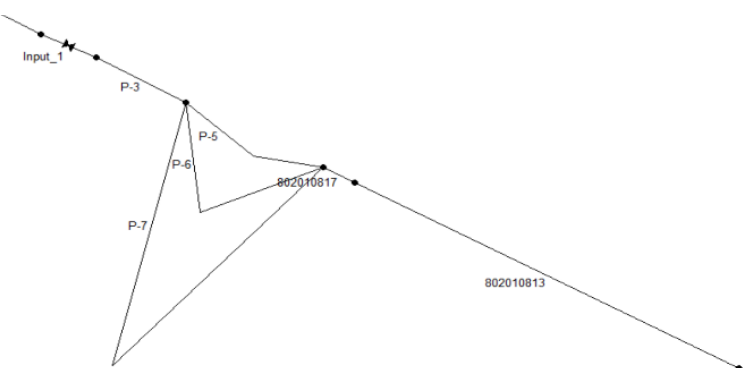

Figure 8 Sample layout for rule controlled parallel pipes with different head-loss coefficient at Input 1

It is possible to calculate the pressure setting of Input 2 by fitting a trend line to the Q-H point cloud. Values under $5 \mathrm{~m}^{3} / \mathrm{h}$ flow rate were excluded from the analysis (coloured light green), because the electromagnetic flow meter installed on the suction side of the PRV is not certified for that low flow conditions. All the values with $0 \mathrm{~m}^{3} / \mathrm{h}$ mean that the valve is fully closed and the pressure on the discharge side is influenced by the other two PRVs supplying the zone. These values are irrelevant when the task is to determine the pressure setting based on operational data. Based on the quadratic trend line the absolute pressure setting of Input 2 was $159.5 \mathrm{~m} \mathrm{H} 2 \mathrm{O}$ over the level of Baltic sea. The point clouds of the other two inputs shows no direct correlation, like Input 2 (Figure 10).

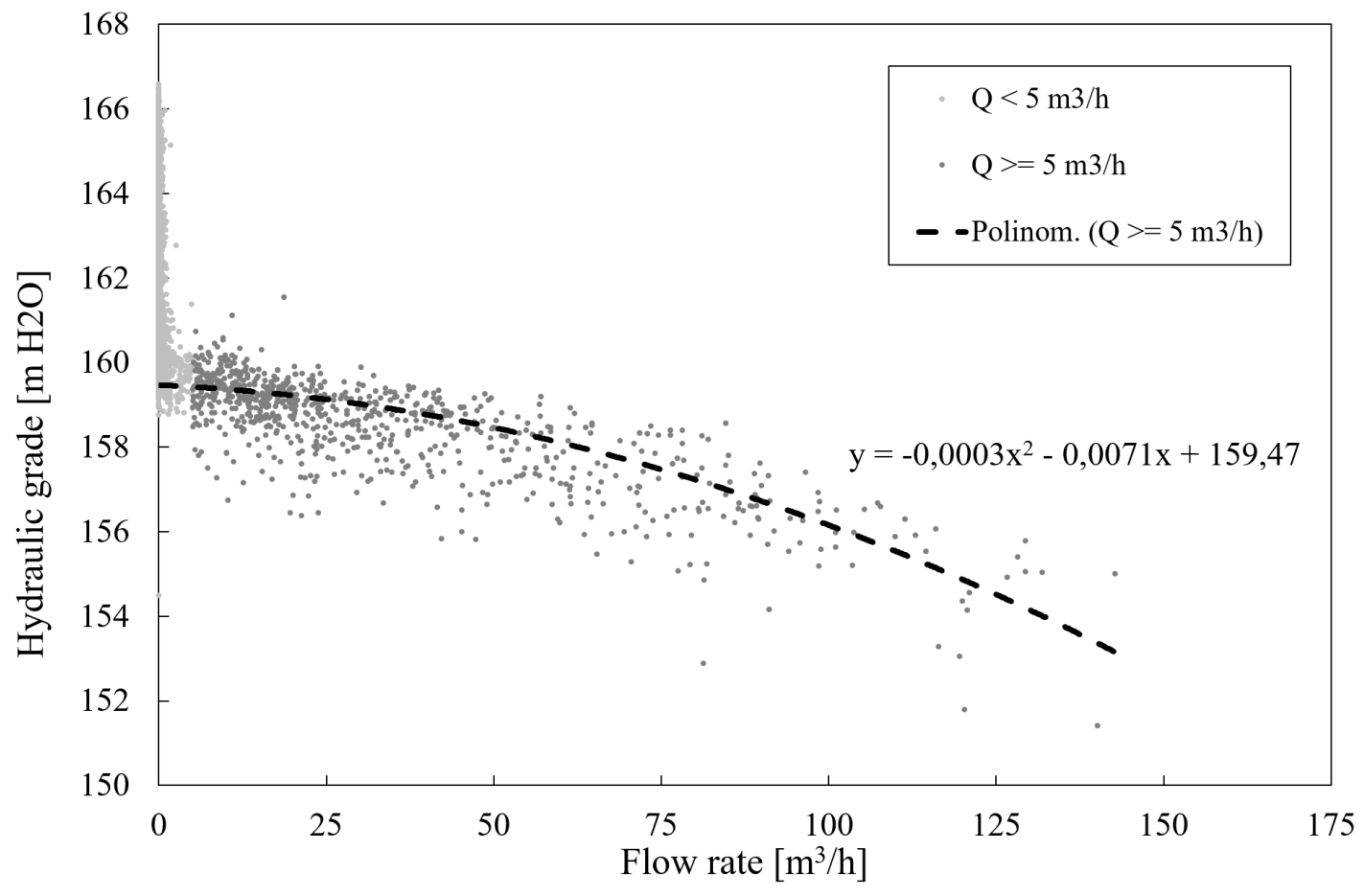

Figure 9 Flow rate - pressure measurements of Input 2

Cluster 3 on Figure 10 shows a disturbance in operation, that caused the pressure to fall $0,4-1,0$ bar below the pressure setting. The $94,8 \%$ of values in cluster 1 occurred between 23:00 and 07:00 and $93,8 \%$ of values in cluster 2 occurred 06:00 to 23:59. The overlapping domain of cluster 1 and cluster 2 in regard of flow rate shows near to equal daily distribution except the evening peak demand (19:00- 
23:00). Note that, the operators did not apply time-based control of pressure settings in the observed timeframe.
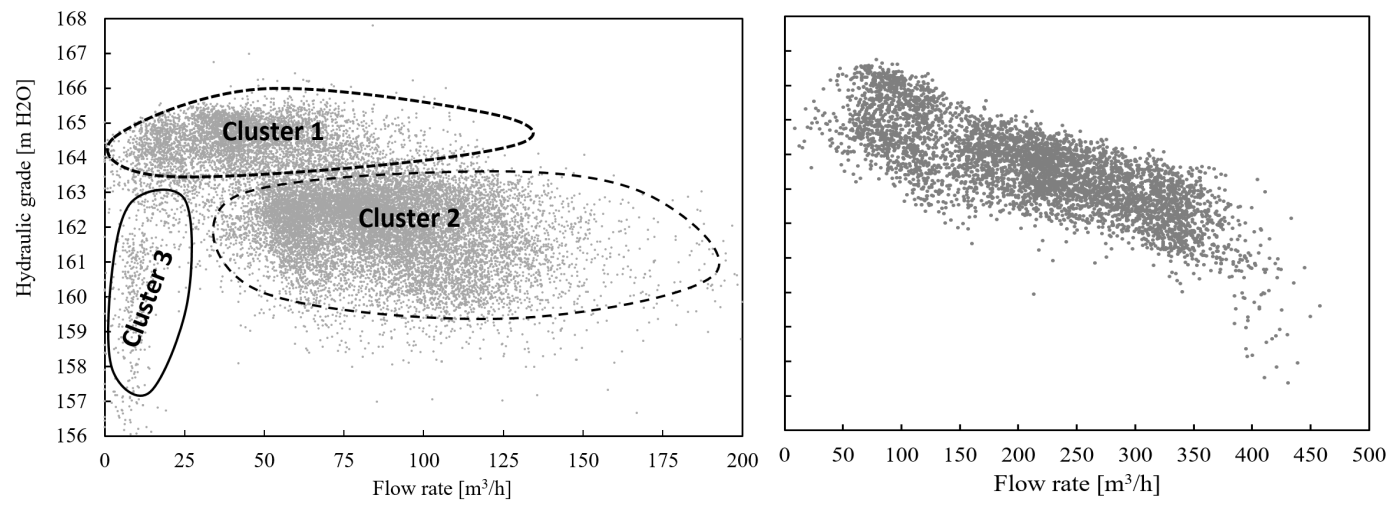

Figure 10 Flow rate - pressure point cloud of Input 3 (left) and Input 1 (right)

After the calibration runs on the modified model, it was possible to achieve better fit on the model to the pressure measurements (Figure 11, Figure 12). The worst fit can be observed when the daily peak demand occurs. This phenomenon correlates with the modified headloss curve shown in Figure 8.
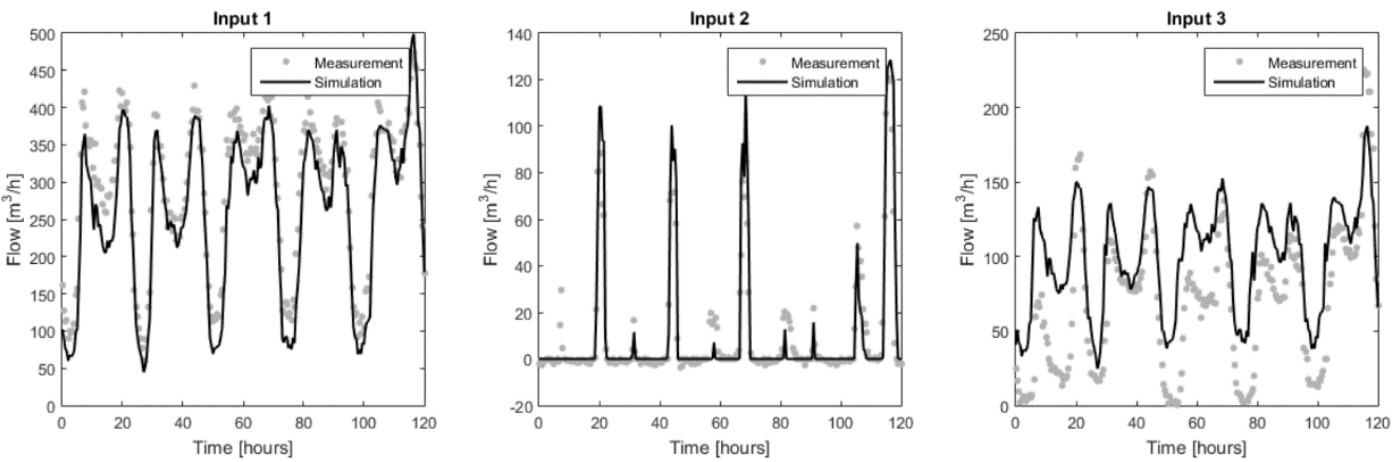

Figure 11 Calibration results in regard to flow measurements with rule-based controls
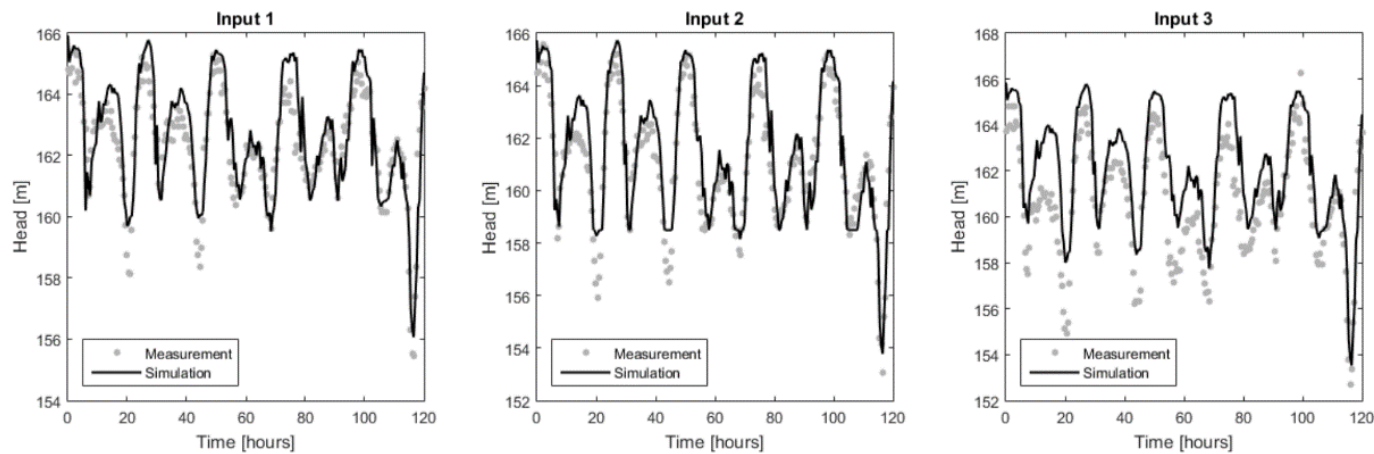

Figure 12 Calibration result in regard to pressure measurements with rule-based controls 


\section{Conclusions}

Both simulated annealing and the Nelder-Mead Simplex method are capable of finding a near-optimal setting to fit the flow rates of PRVs to the measurement time series. Although direct validation of these solutions to the pressure measurement on the downstream side was less successful. The measured pressure losses compared to the valve setting were significantly higher than the simulated pressure losses. The investigation of the pressure loss and flow values showed that calibrating minor loss coefficients will not give a robust solution due to the different pressure loss characteristics of the valve. It is possible to overcome this issue, by applying rule-based controls for parallel links downstream to the PRV, indirectly assigning different head loss coefficients to different domains of the flow range, though the resolution of this solution is limited by the number of parallel pipes downstream the PRV. A robust solution would be the implementation of the possibility of headloss curves into EPANET assigned to the PRVs.

\section{Reference}

[1] A. Knoblock, P. Klingel, E. Oertlé, D. Mutz, P. Fallis, F. Sorg, D. Ziegler, Guidelines for Water Loss Reduction - A Focus on Pressure Management, 2011

[2] L. A. Rossman, EPANET 2 Users Manual, United States Environmental Protection Agency, 2000

[3] T. Janus, B. Ulanicki, Hydraulic modelling for pressure reducing valve controller design addressing disturbance rejection and stability properties, Procedia Engineering 186 (2017) page 635 - 642

[4] Marios K., Demetrios E. "EPANET-Matlab Toolkit" 2010

[5] Lagarias, J. C., J. A. Reeds, M. H. Wright, and P. E. Wright. "Convergence Properties of the NelderMead Simplex Method in Low Dimensions." SIAM Journal of Optimization. Vol. 9, Number 1, 1998, pp. 112-147.

[6] Ingber, L. Adaptive simulated annealing (ASA): Lessons learned. Invited paper to a special issue of the Polish Journal Control and Cybernetics on "Simulated Annealing Applied to Combinatorial Optimization." 1995.

[7] O. Piller, J.E. Van Zyl. Modeling control valves in water distribution systems using a continuous state formulation. Journal of Hydraulic Engineering, American Society of Civil Engineers, 2014, $140(11)$ 